\title{
TRYING TO DO SCIENCE USING HIGH SPECTRAL RESOLUTION SETI PROTOTYPES
}

\author{
Jill Tarter and Peter Backus \\ SETI Institute, 2035 Landings Dr., Mountain View, CA 94043 \\ Kent Cullers, John Dreher, Chris Hlavka and Jane Jordan \\ NASA Ames Research Center \\ Moffett Field, CA 94035-1000
}

Paper Presented by J. Tarter

\begin{abstract}
Poster Paper
Since 1968, a number of observers have attempted to detect a departure from Gaussian amplitude statistics in the radiation from saturated astrophysical masers. In the laboratory, this behavior is exemplified by the mode selection observed in saturated masers. The observed amplitude statistics from astrophysical sources have consistently shown no measurable deviation from Gaussian behavior. Several years ago, a preprint by DeNoyer and Dodd pointed out that all previous measurements were doomed to failure, having been made with frequency resolutions on the order of the Doppler-broadened line widths characteristic of the masers $(\mathrm{kHz})$. They argued that the appropriate resolution for these observations is really that characteristic of the intrinsic coherence width of the maser line (sub- $\mathrm{Hz}$ ), and they reported the results of some initial observations at Arecibo Observatory with $1 / 4-\mathrm{Hz}$ resolution. The results presented in this preprint were inconclusive, but promising. We had the opportunity to make radio frequency interference observations at Arecibo in June, 1989 with two different high resolution SETI prototypes. At that time we used the MCSA 1.0 with $1 / 2-\mathrm{Hz}$ resolution and the upgraded SERENDIP II + with $1-\mathrm{Hz}$ resolution to make long observations of a number of circumstellar and interstellar masers thought to be highly saturated. The initial reduction of data from the MCSA 1.0 has provided a tentatively positive result for one of the features in W51. This poster will discuss these results as well as the data reduction from the SERENDIP II+.
\end{abstract}

